American Journal of Infectious Diseases 3 (2): 76-83, 2007

ISSN 1553-6203

(C) 2007 Science Publications

\title{
The Proteolytic System of Candida dubliniensis
}

\author{
Loaiza-Loeza Salomé, Parra-Ortega Berenice, Bautista-Muñoz Consuelo, Casiano-Rosas César, \\ Hernández-Rodríguez César Hugo, Villa-Tanaca Lourdes \\ Departamento de Microbiología, Escuela Nacional de Ciencias Biológicas \\ Instituto Politécnico Nacional, México, D.F.
}

\begin{abstract}
Proteases of Candida dubliniensis have been scarcely studied, these enzymes may play an important role in nitrogen metabolism, post-translational processing, nutritional stress, dimorphism, virulence, etc. In this work, we report the presence of five different intracellular proteases and one extracellular proteolytic activity. The intracellular proteases are: aminopeptidase ycdAPE, carboxypeptidase ycdCP, dipeptidyl aminopeptidase ycdDAP, proteinases ycdPrA and ycdPrB, and extracellular protease Sap activity, measured under several nutritional conditions. C. dubliniensis produced the highest level of intracellular proteolytic enzymes, i.e., ycdAPE, ycdCP, ycdDAP, ycdPrA and ycdPrB in media with peptone during stationary growth phase. Chelating agents affected mainly APE activity; whereas ycdCp, ycdDAP, and ycdPrB were affected by serine protease inhibitors; ycdPrA was affected by pepstatin, an aspartyl protease inhibitor. We found Sap activity in $C$. dubliniensis in YCB-SBA medium, this activity was inhibited by pepstatin inhibitor. Southern analysis revealed the presence of at least four genes encoding Sap in the $C$. dublinienisis genome (using as probes $S A P 1, S A P 2, S A P 3$, and SAP4-6 genes from C. albicans).
\end{abstract}

Key words: Protease, Sap (secreted aspartyl proteinase), virulence factor, Candida dubliniensis.

\section{INTRODUCTION}

Proteolysis, a vital process for cell life, plays an important role in different physiological functions, such as protein digestion, hormone maturation, immune response, inflammation, fertilization, germination, as well as in other morphological processes. Some proteases are important in spores formation and germination, pathogenesis of several microorganisms, and post-translational regulation ${ }^{[1-3]}$.

Candida dubliniensis was first described as a novel species in 1995 . This organism is very close to the human yeast pathogen, Candida albicans. However, despite the close phylogenetic relationship between $C$. albicans and $C$. dubliniensis, phenotypic and molecular data suggest differences in virulence between these two species. The apparent low virulence of $C$. dubliniensis could be due to differential gene expression rather than to simple absence or divergence of particular genes ${ }^{[4]}$.

The study of Saccharomyces cerevisiae has been important in the elucidation of proteinase multiplicity and proteinase function in eukaryotic cells, numerous proteolytic enzymes have been biochemically and genetically characterized ${ }^{[5]}$. There are several proteases associated with yeast proteasomes that participate in stress-dependent and ubiquitin-mediated proteolysis. They are involved in the degradation of short-lived and regulator proteins that generate small peptides from proteins, these latter are degraded into amino acids by tri-, di-, carboxi-, and amino-peptidases [6-7].

Extracellular proteolytic activity plays a central role in Candida albicans pathogenicity and is produced by a family of 10 secreted aspartyl proteinases (Sap proteins) ${ }^{[3]}$. In vitro, animal and human studies have implicated proteinases in $C$. albicans virulence in one of the following ways: (i) correlation between Sap production in vitro and Candida virulence, (ii) degradation of human proteins and structural analysis in determining Sap substrate specificity, (iii) association of Sap production with other virulence processes of C. albicans, (iv) Sap protein production and Sap immune responses in animal and human infections, (v) SAP gene expression during Candida infections, (vi) modulation of $C$. albicans virulence by aspartyl proteinase inhibitors, and (vii) the use of $S A P$-disrupted mutants to analyze $C$. albicans virulence. Sap proteins fulfill a number of specialized functions during the infective process, which include the simple role of digesting molecules

Corresponding Author: $\quad$ Villa-Tanaca Lourdes, Carpio y Plan de Ayala, s/n, Colonia Santo Tomás, México, D.F.11340 
for nutrient acquisition, digesting or distorting host cell membranes to facilitate adhesion and tissue invasion, and digesting cells and molecules of the host immune system to avoid or resist antimicrobial attack by the host $^{[3]}$.

Detailed analyses of proteases in $C$. dubliniensis might allow us to determine the proteases produced by this fungus and to examine the production of these enzymes in several nutritional conditions and during the dimorphic transition of yeast to mycelia, which is considered to be an important factor in the pathogenicity of this fungus. Moreover, numerous studies have correlated extracellular proteolytic activity in vitro with the virulence of Candida species and have shown that the most virulent species, such as $C$. albicans, C. tropicalis, and $C$. parapsilosis, produce more proteinases in vitro than the less virulent species ${ }^{[3]}$. Secreted aspartic proteinases have been considered a new target in candidiasis therapy ${ }^{[8]}$. In this work, we report the presence of five different intracellular proteases and one extracellular proteolytic activity in $C$. dubliniensis.

\section{MATERIALS AND METHODS}

Strains and growth conditions: Strains $C$. dubliniensis CD36 and C. dubliniensis CD92 were provided by Dr. Derek J. Sullivan of the University of Dublin ${ }^{[9]}, C$. albicans ATTC 10231 was used as reference strain in this study.

The $C$. dubliniensis strains were confirmed to the species level using molecular methods described by Bautista et al. ${ }^{[10]}$. They were routinely maintained on YEPD plates (1\% yeast extract, $2 \%$ peptone, $2 \%$ glucose, and $2 \%$ agar) at $28^{\circ} \mathrm{C}$. The ycdAPE, ycdCP, ycdDAP, ycdPrA and ycdPrB activities in soluble and membrane fractions of $C$. dubliniensis were measured by growing the yeast in YEPD and minimal medium broths containing $0.17 \%$ yeast nitrogen base without amino acids or ammonium sulfate, but adding $2 \%$ glucose with $0.5 \%$ nitrogen source (peptone, proline, or ammonium sulfate) according to manufacturer's instruction. Production of Sap was performed in YCBSBA medium $(1.2 \%$ yeast carbon base, $0.2 \%$ SBA, $\mathrm{pH}$ 4.0 adjusted with $1 \mathrm{M} \mathrm{NaOH}$ ). To evaluate cell growth, absorbance $\left(\mathrm{A}_{600}\right)$ of culture samples was measured in a Perkin-Elmer Lambda IA spectrophotometer.

Preparation of crude extracts and differential centrifugation: Biomass from the culture medium was recovered by centrifugation at $5,000 \mathrm{~g}$ at $4^{\circ} \mathrm{C}$ for 10 min. Cells were fragmented in Braun's mill using glass beads ( $0.5 \mathrm{~mm}$ diameter). The mixture contained $7.5 \mathrm{~g}$

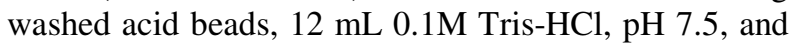
$5 \mathrm{~g}$ cells. Total disintegration time was $10 \mathrm{~min}$ (10 series, 1 min each). The extent of disintegration was monitored under light microscopy. The crude extract was carefully removed from the glass beads and centrifuged at $10,000 \mathrm{~g}$ at $4^{\circ} \mathrm{C}$ for $10 \mathrm{~min}$. The supernatant fluid was removed and centrifuged again at $100,000 \mathrm{~g}$ at $4^{\circ} \mathrm{C}$ for $1.5 \mathrm{~h}$ using a Beckman ultracentrifuge. The $100,000 \mathrm{~g}$ supernatant (S 100,000 $g$ ) was considered as soluble fraction and the membranal fraction was precipitated at 100,000 $g$ (M $100,000 \mathrm{~g}$ ).

Enzyme assays and protein determination: Intracellular enzymatic activities were determined as described by Hirsch et al. ${ }^{[5]}$. The following substrates were used for the different proteases: L-lysyl-4nitroanilide (Lys-4-NA) for aminopeptidase activity (ycdAPE); N-benzoyl-tyrosine-4-nitroanilide (N-BZTyr-4-NA) for carboxypeptidase activity (ycdCP), and L-alanyl-prolyl-4-nitroanilide (Ala-pro-4-NA) for dipeptidyl aminopeptidase activity (ycdDAP), acid denatured hemoglobin for proteinase A activity (ycdPrA), and Hide Powder Azure (HPA) for proteinase B activity (ycdPrB) ${ }^{[11]}$. Assay for Sap activity was based on the MacDonald and Odds [12] assay, enzyme activity was measured spectrophotometrically following digestion of BSA as substrate. A typical reaction mix containing $500 \mu \mathrm{l}$ $2.0 \%(\mathrm{w} / \mathrm{v}) \mathrm{BSA}$ in $50 \mathrm{mM}$ sodium citrate (pH 3.2), 100 $\mu \mathrm{l} 50 \mathrm{mM}$ sodium citrate $(\mathrm{pH} 3.2)$, and $200 \mu \mathrm{l}$ of culture supernatant was incubated for $30 \mathrm{~min}$ at $37^{\circ} \mathrm{C}$. The reaction was stopped by adding $200 \mu \mathrm{l} 10 \%$ TCA, precipitated protein was removed by centrifugation at $22,000 \mathrm{~g}$ for $5 \mathrm{~min}, 75 \mu \mathrm{l}$ of the supernatant was removed and added to $75 \mu \mathrm{l} 0.5 \mathrm{M} \mathrm{NaOH}$. Tyrosinecontaining peptides in the neutralized sample were determined with the Folin's reagent according to the method of Lowry. Protein determinations were performed according to the Bradford method ${ }^{[13]}$.

Effect of protease inhibitors on proteolityc activities: The effect of protease inhibitors (leupeptin, pefabloc, E-64, EDTA, pepstatin, 1-10, phenanthroline, and PMSF) on the proteolytic activities was measured in $100 \mathrm{mM}$ Tris- $\mathrm{HCl}$ buffer, $\mathrm{pH}$ 7.5. The soluble fraction $(100,000 \mathrm{~g})$ or the culture medium's supernatant, after harvesting cells (for Sap activity), was preincubated with the respective compound for $30 \mathrm{~min}$ at $37^{\circ} \mathrm{C}$, followed by the standard enzyme assay. 
Southern hybridization: Genomic DNA from $C$. dubliniensis was digested with EcoRI endonuclease, subsequently separated by electrophoresis in agarose and transferred to a Nylon membrane positively charged (Amersham Pharmacia Biotech, UK), as described by Sambrook et al. ${ }^{[14]}$. Hybridization was performed at $65^{\circ} \mathrm{C}$ with $S A P 1, S A P 2 S A P 3$, or SAP4-6 specific probes labeled with digoxigenin-dUTP (20deoxyuridine 50-triphosphate) using a random-primed digoxigenin (DIG) DNA labeling detection kit (Roche, Mannheim, Germany). Hybridization and immunological detection were performed as recommended by the supplier. SAP DNA probes to be used in hybridization experiments were generated for PCR from C. albicans SAP genes: SAP1, SAP2, SAP3, or SAP4-6 as described by Bautista-Muñoz et al. ${ }^{[10]}$. Genomic DNA from C. albicans ATCC 10231 was used as template.

\section{RESULTS}

ycdAPE, ycdCP, ycdDAP, ycdPrA, ycdPrB, and Sap proteolytic activities under different nitrogen sources: $\quad C$. dubliniensis presented ycdAPE, ycdDAP, ycdCP, ycdPrA and ycdPrB intracellular activities. The highest levels of the three exopeptidases ycdAPE, ycdDAP, ycdCP proteolytic enzymes were reached in the medium enriched with peptone as nitrogen source: YPD and YNB-peptone, Table 1. These intracellular exopeptidases were accumulated during the stationary phase $(48 \mathrm{~h})$. All of them were localized in the soluble fraction (S 100,000 $g$ ), Table 2. The highest level of intracellular acidic proteinase ycdPrA was reached in the medium with amonium as nitrogen source during the logarithmic phase $(6 \mathrm{~h})$, Table 1 . This activity was localized in the soluble fraction (S 100,000 $\mathrm{g}$ ) although considerable ycdPrA activity was also found in the membrane fraction (M 100,000 g), Table 2. The production of proteinase $\mathrm{ycdPrB}$ was regulated by the nitrogen source, the highest level of this proteinase was reached in the medium with peptone and proline during early stationary phase, Table 1 . This activity was localized in the soluble fraction (S 100,000 g), Table 2. It was not possible to detect Sap activity in YPD and YNBdifferent nitrogen sources media. The medium YCBSBA has been described as inductor medium for the production of Sap in C. albicans, this medium was also

an inductor for the production of C. dubliniensis Sap activity. There was no difference in the level of Sap activity between $C$. dubliniensis and C. albicans, Fig. 1 .

Effect of inhibitors on enzymes activity: The effect of seven inhibitors was tested in the $\mathrm{S} 100,000 \mathrm{~g}$ fraction (YPD medium) with ycdAPE, ycdDAP, ycdCP, ycdPrA and $y c d P r B$, as well as in the supernatant of the culture medium (YCB-SBA) after harvesting cells with Sap activity. Chelating agents, such as EDTA and 1-10 phenanthroline, exerted a clear inhibitory effect on ycdAPE activity, suggesting that this protease is a metalloenzyme. PMSF, pefabloc (serine protease inhibitors) caused inhibition of the three enzymes (ycdAPE, ycdDAP, and ycdCP). E-64 (cysteine proteases inhibitor) had the same effect on ycdAPE activity; however, leupeptin (cysteine and serine proteases inhibitors) only inhibited ycdPrB, Table 3. Acidic proteinases ycdPrA and Sap were inhibited by pepstatin (aspartyl protease inhibitor). Results suggest that ycdAPE is a metallo-serin protease; ycdDAP, ycdCP and ycdPrB are serine proteases; and ycdPrA and Sap are aspartyl proteases.

A)

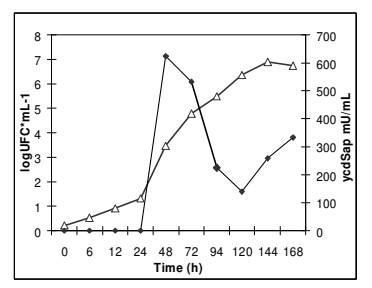

C)

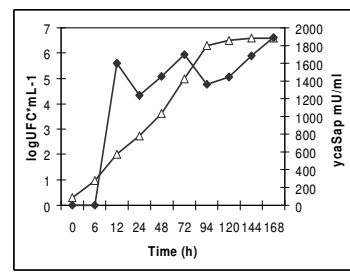

Fig1. Kinetic study of Sap production by $C$. dubliniensis and $C$. albicans in different media. A) ycdSap in YCB-SBA medium. B) ycdSap in YPD medium. C) ycaSap in YCB-SBA medium. D) ycaSap in YPD medium. Total activity $(\bullet)$, Growth $(\triangle)$.

Presence of $S A P$ genes in $C$. dubliniensis genome: $C$. dubliniensis presented extracellular aspartyl proteinase activity with the inductor medium YCB-SBA. We 
therefore decided to examine $C$. dubliniensis for the presence of homologues of the known $C$. albicans SAP genes, using probes derived from six members of the SAP multigene family (SAP1, SAP2, SAP3, SAP4-6). Genomic DNA from $C$. dubliniensis CD36 and CD92 isolates was digested with EcoRI endonuclease and Southern transfered to nylon membrane after gel electrophoresis. C. dubliniensis CD36 and CD92 gave identical hybridizing fragments for $S A P 1, S A P 2, S A P 3$,
SAP4-6 probes, Fig. 2. The restriction fragment sizes (band hybridizing) to probe $S A P l$ were: 4.8 and $4.0 \mathrm{~kb}$; to probe $S A P 2: 4.8,4.0$, and $1.4 \mathrm{~kb}$; to probe $S A P 3: 4.8$ $\mathrm{kb}$; and to probes SAP4-6: $3.3 \mathrm{~kb}$. These results confirm the similitude that exists between nucleotidic sequences of $S A P 1, S A P 2$, and $S A P 3$ genes. However, only one band hybridizing to probe SAP4-6 was revealed in the C. dubliniensis genome.

Table 1: Proteolytic enzyme activities in soluble cellular fraction of $C$. dubliniensis

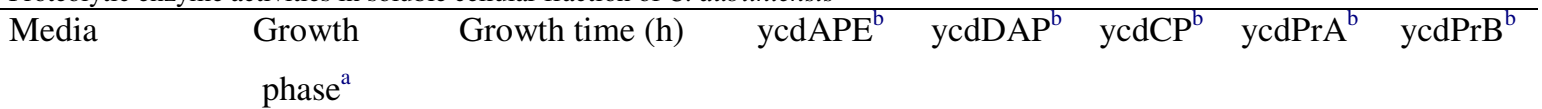

Specific activity ( $\mathrm{mU} / \mathrm{mg}$ of protein)

\begin{tabular}{cccccccc}
\hline YPD & I & 12 & 117.54 & 3.15 & 4 & 0.35 & 3.02 \\
YNB-peptone & II & 24 & 174.72 & 4.3 & 11.38 & 0.53 & 8.1 \\
& III & 48 & 177.76 & 7.0 & 18.04 & 0.45 & 8.9 \\
YNB-proline & I & 6 & 3.23 & 0.72 & 0.7 & 0.06 & 3.18 \\
& II & 18 & 7.65 & 0.67 & 2.48 & 0.07 & 7.46 \\
& III & 48 & 5.22 & 1.08 & 3.36 & 0.05 & 0.0 \\
YNB-ammonium & I & 6 & 2.69 & 0.66 & 0.73 & 0.04 & 0.85 \\
& II & 18 & 1.63 & 0.24 & 2.14 & 0.14 & 10.75 \\
& III & 48 & 3.12 & 0.81 & 2.85 & 0.07 & 0.41 \\
& I & 6 & 3.01 & 0.68 & 0.42 & 5.08 & 2.78 \\
& II & 18 & 6.01 & 0.47 & 2.39 & 0.13 & 1.52 \\
& III & 48 & 0.94 & 0.0 & 0.36 & 0.18 & 1.72
\end{tabular}

${ }^{\mathrm{a} E x p o n e n t i a l}$ (I), early stationary phases (II), late stationary phases (II).

${ }^{b}$ Cellular fractions were prepared from cells grown in the corresponding medium. The 100,000 $g$ soluble fraction was used to determine ycdAPE, ycdCP, ycdDAP, ycdPrA and ycdPrB enzymatic activities against Lys-4-NA, Ala-Pro-4-NA, N-Bz-Tyr-4-NA, acid-denatured hemoglobin and HPA.

Table 2: Distribution of enzyme activities in cellular fractions of $C$. dubliniensis

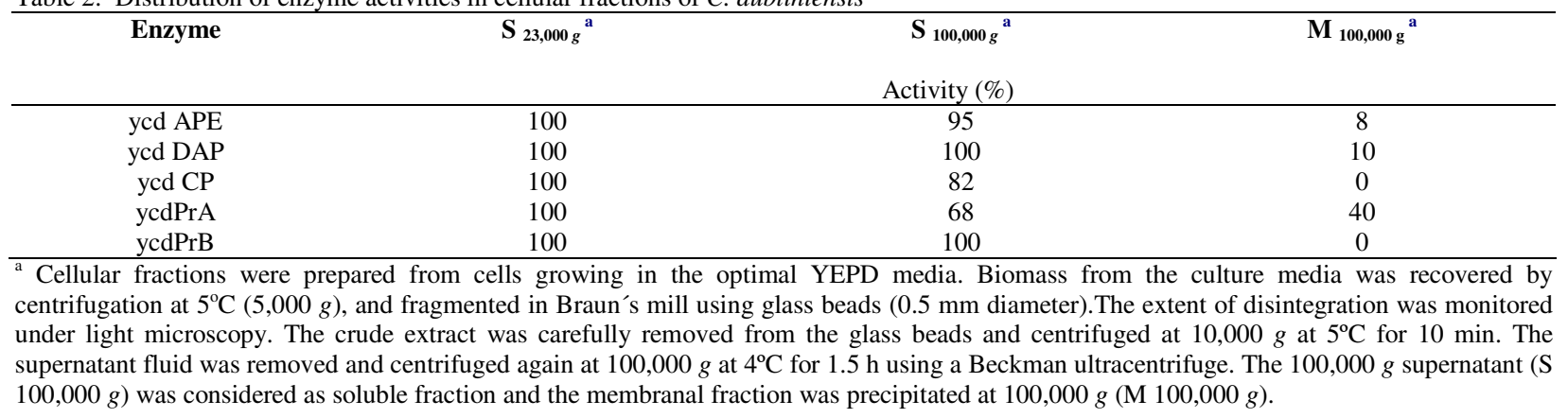


Am. J. Infect. Dis., 3 (2): 76-83, 2007

Table 3: Effect of specific inhibitors of proteases on the activity of the intracellular proteases ycdAPE, ycdCP, ycdDAP, ycdPrA and ycdPrB and extracellular Sap activity from C. dubliniensis

\begin{tabular}{|c|c|c|c|c|c|c|c|}
\hline \multirow[t]{2}{*}{ Inhibitors } & \multirow[t]{2}{*}{ Concentration } & ycdAPE & ycdCP & ycdDAP & ycdPrA & ycdPrB & ycdSap \\
\hline & & \multicolumn{6}{|c|}{ Residual activity $^{a}$} \\
\hline \multirow[t]{2}{*}{ EDTA } & $1(\mathrm{mM})$ & 49.37 & 100 & 85.5 & 95 & 100 & 98 \\
\hline & $10(\mathrm{mM})$ & 3.74 & 88 & 60 & 54.5 & 66 & 80 \\
\hline \multirow[t]{2}{*}{ 1-10 Phenantroline } & $1(\mathrm{mM})$ & 40.12 & 83.5 & 80 & $\mathrm{nd}^{\mathrm{b}}$ & 55 & 89 \\
\hline & $10(\mathrm{mM})$ & 11.87 & 66 & 71 & $\mathrm{nd}^{\mathrm{b}}$ & 42 & 82 \\
\hline \multirow[t]{2}{*}{ PMSF } & $1(\mathrm{mM})$ & 33.46 & 12 & 14 & 97 & 46 & 92 \\
\hline & $5(\mathrm{mM})$ & 9.97 & 8 & 8 & 80 & 40 & 85 \\
\hline \multirow[t]{2}{*}{ E-64 } & $10(\mu \mathrm{M})$ & 43.34 & 82 & 65 & 94 & 100 & 95 \\
\hline & $50(\mu \mathrm{M})$ & 15.46 & 66 & 60 & 93 & 52 & 92 \\
\hline \multirow[t]{2}{*}{ Leupeptine } & $20(\mu \mathrm{M})$ & 88.32 & 100 & 100 & 100 & 44 & 100 \\
\hline & $50(\mu \mathrm{M})$ & 81.2 & 95 & 87 & 94 & 33 & 93 \\
\hline \multirow[t]{2}{*}{ Pefabloc } & $1(\mathrm{mM})$ & 32.1 & 92.5 & 62 & $\mathrm{nd}^{\mathrm{b}}$ & 52 & 95 \\
\hline & $5(\mathrm{Mm})$ & 16.45 & 91.4 & 53 & $\mathrm{nd}^{\mathrm{b}}$ & 49 & 94 \\
\hline \multirow[t]{2}{*}{ Pepstatin A } & $2.5(\mu \mathrm{M})$ & $\mathrm{nd}^{\mathrm{b}}$ & $\mathrm{nd}^{\mathrm{b}}$ & $\mathrm{nd}^{\mathrm{b}}$ & 25 & $\mathrm{nd}^{\mathrm{b}}$ & 20 \\
\hline & $25(\mu \mathrm{M})$ & $\mathrm{nd}^{\mathrm{b}}$ & $\mathrm{nd}^{\mathrm{b}}$ & $\mathrm{nd}^{\mathrm{b}}$ & 14 & $\mathrm{nd}^{\mathrm{b}}$ & 12 \\
\hline
\end{tabular}

${ }^{a}$ Expressed as a percentage of the activity obtained in the absence of any added inhibitor, which was assigned a value of $100 \%$.

${ }^{\mathrm{b}}$ nd: non determined.

\section{DISCUSSION}

This paper presents, as far as we know, the first report on intracellular proteases of $C$. dubliniensis and suggests four encoding SAP genes on the genome of this CNCA (Candida no C. albicans). Proteolysis plays an important role in different physiological functions of the cell and the recently discovered secreted aspartic proteinase multi-gene (SAP) family in C. albicans has complicated assessment of proteolytic activity as a factor in the onset and development of Candida infections.

Our finding demonstrates that C. dubliniensis expresses intracellular proteolytic activities similar to those found in other yeasts, such as $S$. cerevisiae, Schizosaccharomyces pombe, Kluyveromyces lactis, and Yarrowia lipolytica ${ }^{[15-19]}$. There are not many reports on intracellular proteases in fungi pathogenic to humans. The obtained data revealed that proteases ycdAPE, ycdDAP, ycdCP, ycdPrA, and ycdPrB might be regulated by nitrogen and carbon sources, as happens in other fungi ${ }^{[20-22,2,18,11]}$.

C. dubliniensis presented a soluble ycdAPE activity, this activity seems to be a metalloprotease, according to the results obtained in the inhibition assays. This inhibitory effect has been observed in other aminopeptidases isolated from yeasts, such as AP-Y from $S$. cerevisiae ${ }^{[23]}$, aminopeptidase I from $S$. pombe ${ }^{[24]}$, lysine aminopeptidase from Kluyveromyces marxianus ${ }^{[25]}$, lysine aminopeptidase from $Y$. lipolytica ${ }^{[19]}$. The presence of blocking agents from serine and cysteine proteases, such as pefabloc and PMSF, inhibited the activity, suggesting that serine residues and thiol groups might be participating in the catalysis of this enzyme. Most of the aminopeptidases described in lactic bacteria are metalloproteases that have a very similar inhibition pattern to that of the aminopeptidase from C. dubliniensis [26-27]. An immunogenic aminopeptidase of $C$. albicans has been described as a metallopetidase of $52 \mathrm{kDa}$ and has been proposed for the diagnosis of systemic candidiasis ${ }^{[28]}$. 
Two carboxypeptidases have been described mainly in $S$. cerevisiae: yscCPY and yscCPS ${ }^{[29]}$. We detected one carboxypeptidase activity that was located in the soluble fraction; ycdCP activity in $C$. dubliniensis is associated with an endogenous inhibitor, since it was necessary to incubate the extracts with $0.5 \%$ sodium deoxycolate to detect the activity of this enzyme ${ }^{[16]}$ (data not shown). ycdCP activity seems to be a serine protease. CYP1 C. albicans gene encodes a protein with 74\% identity with CYP from $S$. cerevisiae. Reports on carboxypeptidase actvities in $C$. albicans are scarce; howewer, an extracellular carboxyl proteinase produced by the yeast $C$. albicans enhanced vascular permeability when injected into the dorsal skin of guinea pigs ${ }^{[30]}$.
A
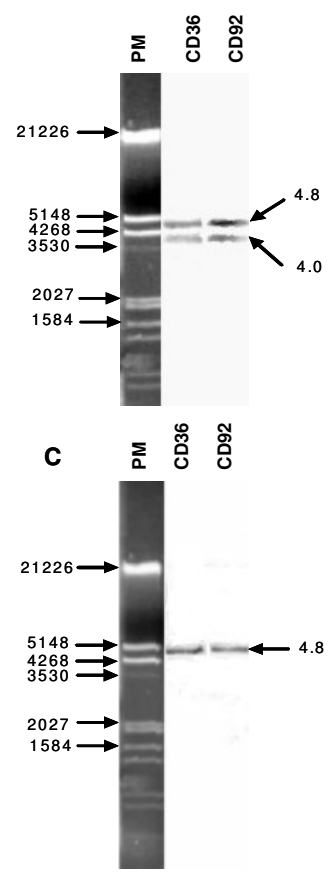

B
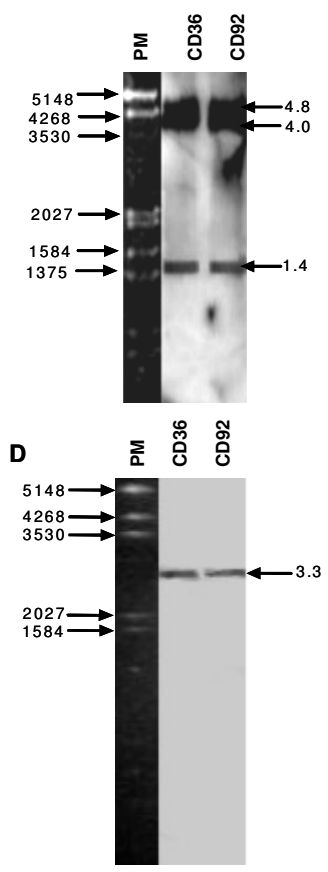

Fig. 2: Southern blots analysis of EcoRI digestions of C. dubliniensis CD36 and C. dubliniensis CD92 that were hybridized with the SAP1 (Panel A), SAP2 (Panel B), SAP3 (Panel C) and SAP4-6 (Panel D) genes from $C$. albicans. The molecular size markers ( $\mathrm{pb})$ are indicated to the left. The genes found in CD36 and CD92 are indicated to the rigth $(\mathrm{kb})$.

We detected a ycdDAP activity localized in the soluble fraction. The activity of ycdDAP was inhibited by E-64, PMSF, and pefabloc, indicating that it is probably a serine protease. It is interesting to note that the dipeptidyl aminopeptidase of $S$. cerevisiae, called yscIV, is a membrane serine exopeptidase involved in the processing of the $\alpha$-sexual factor ${ }^{[29]}$. A dipeptidylaminopeptidase encoded in the STE13 gene has been proposed in $C$. albicans that could participate in the processing of a putative $\alpha$-sexual factor or of other peptides ${ }^{[31]}$. The ycdDAP found in $C$. dubiniensis might correspond to the activity that participates in controlling post-translational processing of other peptides.

An acidic proteinase intracellular activity was detected in the soluble fraction (S 100,000 $\mathrm{g}$ ). This ycdPrA activity is an aspartyl proteinase (inhibited by pepstatin). Probably this activity is similar to yscA from $S$. cerevisiae, a soluble vacuolar endoprotease that participates in protein degradation, nitrogen metabolism, acting like a vacuolar hydrolase, and precursor proccesing ${ }^{[29]}$. In other yeasts, like $C$. albicans, $S$. pombe, K. lactis, and Ustilago maydis, an intracellular acidic aspartyl $\operatorname{PrA}$, inhibited by pepstatin ${ }^{[2,18,11]}$ has also been described.

The ycdPrB activity was detected in the soluble fraction (S 100,000 $\mathrm{g}$ ) and seems to be a metallo-serinecysteine protease. In $S$. cerevisiae, the gene that encodes vacuolar proteinase yscB activity is regulated by nitrogen and carbon sources, and depends on the growth phase ${ }^{[32]}$. In turn, $K$. lactis produces a yklB similar to that detected in $S$. cerevisiae ${ }^{[18]}$. Likewise, it is known that yeasts $S$. pombe and $C$. albicans produce proteinase $\mathrm{B}$ activities called yspB and ycaB, respectively ${ }^{[2]}$.

In $S$. cerevisiae, yscB participates in the proteolytic processing during maturation of other enzymes ${ }^{[29]}$. The role played by the ycdPrB detected in C. dubliniesis must still be elucidated. In Saccharomyces carlbergensis, proteinase B activity is regulated by an endogenous inhibitor as occurs in S. cerevisiae ${ }^{[33]}$.

The studied yeast presents Sap activity that is associated to the logarithmic growth phase, and the enzymatic levels are higher when a protein, such as albumin, provides the nitrogen source. Similarly to the Sap described for $C$. albicans, the C. dubliniensis Sap is synthesized when the initial $\mathrm{pH}$ of the medium is acidic (3.0-5.0), suggesting the relation of one or more aspartyl proteinases, considering that in other Candida species more than one Sap activity have been found ${ }^{[34]}$. In $C$. albicans, the sequences of each of the SAPISAP10 genes described up to now are highly homologous among them ${ }^{[35]}$. Southern analysis, using heterologous probes of $C$. albicans for genes $S A P 1$, SAP2, SAP3, SAP4-SAP6, revealed hybridization bands of different molecular size for the $S A P$ genes in the $C$. dubliniensis genome. Since the sequence of the genome of this yeast is not known yet, we can only assure that C. dubliniensis possesses four homologous sequences 
to those of the $S A P$ genes described in $C$. albicans. Most probably the $4.0 \mathrm{~kb}$ hybridization band is the homologous of the $S A P 1$ gene of $C$. albicans; the $1.4 \mathrm{~kb}$ hybridization band would correspond to the $S A P 2$ gene; the $4.8 \mathrm{~kb}$ band would be homologous to the SAP3 gene. The single $3.3 \mathrm{~kb}$ hybridization band would correspond to any of the three $S A P$ genes (SAP4, SAP5, $S A P 6)$ of $C$. albicans. Currently, our group is working on the deduced nucleotidic and amino-acidic sequence of these four genes.

Gilfillan et al. [36] reported the presence of homologue sequences to the SAP genes described in $C$. albicans, through Southern analysis of chromosomes separated by PFGE and hybridization of heterologous probes of SAP1, SAP2, SAP3, SAP5, and SAP7. Since genes $S A P 4, S A P 5$, and SAP6 were $90 \%$ identical and could not be distinguished through hybridization with specific probes for each one, the gene SAP5 was used as probe to search for the presence of SAP4-SAP5SAP6.

C. dubliniensis is an attractive model to study the production of proteases because it is a recently identified species that is implicated in oral candidosis in HIV-infected and AIDS patients. Numerous reports have described a significant increase in the incidence and diagnosis of opportunistic and systemic candidosis during the last decade ${ }^{[37]}$. The $C$. dubliniensis species shares many phenotypic characteristics with and is closely related phylogenetically to $C$. albicans. Many studies with $C$. albicans have implicated the Sap proteinases in virulence; hence, it will be necessary to study the role played by these $C$. dubliniensis proteases.

\section{ACKNOWLEDGEMENTS}

This study was supported by research grant from CONACYT-SALUD-14299; SIP-IPN 20070248 and SIP-IPN 20060442. B. Parra-Ortega and C. BautistaMuñoz are CONACyT fellows, C. Casiano-Rosas was PIFI-IPN fellow and S. Loaiza-Loeza is IPN and PROMEP fellow. C. Hernández-Rodríguez and L. Villa-Tanaca received COFAA-IPN, EDI-IPN and SNI supports.

\section{REFERENCES}

1. Yuan, L. and G.T. Cole, 1989. Characterization of proteinase inhibitors isolated from fungal pathogen. Coccidiodes immitis. J. Biochem., 257: 729-736.

2. Suárez-Rendueles, P, L. Villa and M.J. Arbesú et al., 1991.The proteolytic system of the fission yeast Schizosaccharomyces pombe. FEMS Microbiol. Lett., 81: 215-220.
3. Naglik, J.R., S.J. Challacombe and B. Hube, 2003. Candida albicans secreted aspartyl proteinases in virulence and pathogenesis. Microbiol. Mol. Biol., 67: 400-428.

4. Sullivan, D.J., G.P. Moran and D.C. Coleman, 2005. Candida dubliniensis: Ten years on. FEMS Microbiol. Lett., 253: 9-17.

5. Hirsch, H. H., P. Suárez-Rendueles and D.H. Wolf, 1989. Yeast (Saccharomyces cerevisiae) proteinases: Structure, characteristics and functions. In: The molecular and cell Biology of yeast (eds E.F. Walton and G.T.Yaranton) pp. 134200. Blackie \& Sons Ltd, London.

6. Hilt, W. and D.H. Wolf, 1992. Stress-induced proteolysis in yeast. Mol. Microbiol., 6: 24372442.

7. Kisselev, A.F., T.N Acopian and K.M Woo et al., 1999. The sizes of peptides generated from protein by mammalian 26 and $20 \mathrm{~S}$ proteasomes. Implications for understanding the degradative mechanism and antigen presentation. J. Biol. Chem., 274: 3363-3371.

8. Bein, M., M. Schaller and H.C. Kortig, 2002. The secreted aspartic proteinases as a new target in the therapy of candidiasis. Curr. Drug Targets, 3: 5157.

9. Sullivan, D.J., T.J. Westerneng and K.A. Haynes et al., 1995. Candida dubliniensis sp. nov.: phenotypic and molecular characterization of a novel species associated with oral candidosis in HIV-infected individuals. Microbiology, 141: 1507-1521.

10. Bautista-Muñoz, C., X.M. Boldo and L. VillaTanaca et al., 2003. Identification of Candida sp by randomly amplified polymorphic DNA analysis and differentiation between Candida albicans and Candida dubliniensis by direct PCR methods. J. Clin. Microbiol., 41: 414-420.

11. Mercado Flores, Y, C.H. Hernández-Rodríguez and J. Ruiz-Herrera et al., 2003. Proteinases and exopeptidases from the phytopathogenic fungus Ustilago maydis. Mycologia, 95: 327-339.

12. MacDonald, F. and F.C. Odds, 1980. Inducible proteinase of Candida albicans in diagnostic serology and in the pathogenesis of systemic candidosis. J. Med. Microbiol., 13: 423-535.

13. Bradford, M., 1976. A rapid and sensitive method for the quantitation of microgram quantities of protein utilizing the principle of protein-dye binding. Anal. Biochem., 72: 248-254.

14. Sambrook, J., E.F. Fritsch and T. Maniatis, 1989. Molecular Cloning: A Laboratory Manual, $2^{\text {nd }}$ edn. Cold Spring Harbor Laboratory, Cold Spring Harbor, NY. 
15. Wolf, D.H. and U. Weiser, 1977. Studies on a carboxypeptidase $\mathrm{Y}$ mutant of yeast and evidence for a second carboxypeptidase activity. Eur. J. Biochem., 73: 553-556.

16. Wolf, D.H. and C. Ehmann, 1981. Carboxypeptidase $\mathrm{S}$ and carboxypeptidase $\mathrm{Y}$ deficient mutants of Saccharomyces cerevisiae. J. Bacteriol., 147: 418-426.

17. Spormann, D.O., J. Heim and D.H. Wolf, 1991. Carboxypeptidase yscS gene structure and function of the vacuolar enzyme. Eur. J. Biochem., 197: 399-405.

18. Flores, M.V., A. Cuellas and C.E. Voget, 1999. The proteolytic system of the yeast Kluyveromyces lactis. Yeast, 15: 1437-1448.

19. Hernández-Montañez, Z., J. Araujo-Osorio and Y. Noriega-Reyes et al., 2007. The Intracellular Proteolytic System of Yarrowia lipolytica and characterization of an aminopeptidase. FEMS Microbiol. Lett., 268: 178-186.

20. Hansen, R.J., R.L. Switzer and H. Hinze et al., 1977. Effect of glucose and nitrogen source on the levels of proteinases, peptidases and proteinase inhibitors in yeast. Biochim. Biophys. Acta., 496: 103-114.

21. Wolf, D.H. and H. Holzer, 1980. Proteolysis in yeast. In: Microorganisms and nitrogen sources (eds J.W. Payne and J.W) pp. 431-458. John Wiley.22. Park, J.S., E.S. Hwang and S.N. Park et al., 1997. Physical status and expression of HPV in cervical cancers. Gynecol. Oncol., 65: 121-129.

22. García-Ramos, C, R. Cruz-Camarillo and S. Gascon et al., 1983. Studies on the regulation of $\mathrm{x}-$ prolyl dipeptidyl aminopeptidase activity. J. Gen. Microbiol., 129: 3519-3523.

23. Yasuhara, T., T., Nakai and A. Ohashi, 1994. Aminopeptidase Y, a new aminopeptidase from Saccharomyces cerevisiae. J. Biol. Chem., 269: 13644-13650.

24. Arbesú, M.J., E. Valle and P. Suárez-Rendueles, 1993. Purification and characterization of aminopeptidase from Schizosaccharomyces pombe. Yeast, 12: 1-16.

25. Ramírez-Zavala B, Y. Mercado-Flores and C. Hernández-Rodríguez C et al., 2004. Purification and characterization of a lysine aminopeptidase from Kluyveromyces marxianus. FEMS Microbiol. Lett., 235: 369-375.

26. Fernández J., A.F. Mohedano and P. Gaya et al., 2000. Purification and properties of two intracellular aminopeptidases produced by Brevibacterium linens SR3. Int. Dairy. J., 10: 241248.
27. Magboul, A.A.A. and P.L.H McSweeney, 1999. Purification and characterization of an aminopeptidase from Lactobacillus curvatus DPC2024. Int. Diary. J., 9: 107-116.

28. El Moudini B., M.H. Rodier and G. Daniault et al., 1998. Improved immunodiagnosis of human candidiasis by an enzyme-linked immunosorbent assay using a Candida albicans 52-kilodalton metallopeptidase. Clin. Diagn. Lab. Immunol., 5: 823-825.

29. Suárez-Rendueles, P. and D.H Wolf, 1988. Proteinase function in yeast biochemical and genetic approaches to a central mechanism of posttranslational control in the eukariotic cell. FEMS Microbiol. Lett., 54: 17-46.

30. Kaminishi H.M, M.Tanaka and T. Cho et al., 1990. Activation of the plasma kallikrein-kinin system by Candida albicans proteinase. Infect. Immun., 58: 2139-2143.

31. Bautista-Muñoz, C., C. Hernández-Rodríguez and L.Villa-Tanaca, 2005. Analysis and expression of STE13ca gene encoding a putative X-prolyldipeptidyl aminopeptidase from Candida albicans. FEMS Immunol. Med. Microbiol., 45: 459-469.

32. Naik, R.R. and E.W. Jones, 1997. Regulation of the proteinase B structural gene PRB1 in Saccharomyces cerevisiae. J. Bacteriol., 179: 14691474.

33. Matern, H., U. Weiser and H. Holzer, 1979. Isolation, characterization and localization of the proteinase B inhibitor IB3 from Saccharomyces carlsbergensis. Eur. J. Biochem., 101: 325-332.

34. De Bernardis, F., P.A. Sullivan and A. Cassone, 2001. Aspartyl proteinases of Candida albicans their role in pathogenicity. Med. Mycol., 39: 303313.

35. Monod, M., G. Togni and D. Sanglard, 1994. Multiplicity of genes encoding secreted aspartic proteinases in Candida species. Mol. Microbiol., 13: 357-368.

36. Gilfillan G., D.J. Sullivan and K. Haynes et al., 1998. Candida dubliniensis: phylogeny and putative virulence factors. Microbiology, 144: 829838.

37. Moran, G.P., D.J. Sullivan and D.C. Coleman, 2002. Emergence of Non-Candida albicans Candida species as pathogen. In: Candida and candidiasis. (eds R.A. Calderone) pp. 37-53. ASM Press. EUA. 